\title{
Perfil de pacientes atendidos no ambulatório de nutrição de uma Clínica Escola em uma Universidade particular de São Luís - MA
}

\section{Profile of Patients Served in the Nutrition Ambulatory of a School Clinic in a Private University of São Luís - MA.}

Vanessa Fernanda Gomes de Brito Pereira ${ }^{1}$, Rakel de Sousa Oliveira Mendes², Luciana Pereira Pinto Dias ${ }^{3}$, Lívia Muritiba Pereira de Lima Coimbra 4

\section{RESUMO}

É evidente o crescente número de doenças crônicas não transmissíveis na população. Dessa forma, a procura por atendimento nutricional tem aumentado. O estudo teve como objetivo identificar o perfil dos pacientes atendidos no ambulatório de nutrição de uma Clínica escola em uma Universidade particular de São Luís - MA. Trata-se de um estudo transversal com coleta de dados secundária ocorrida no período de agosto a setembro de 2019. Foram analisados 85 prontuários do ano de 2018 de pacientes de ambos os sexos e de todas as idades, sendo excluídos prontuários com informações incompletas pertinentes a realização da pesquisa. Os dados foram transcritos para um protocolo previamente elaborado. Dos 85 prontuários avaliados, verificou-se que $54,1 \%$ dos pacientes eram adultos. A maior procura pelo atendimento nutricional foi do gênero feminino $(74,1 \%)$. De acordo com os hábitos de vida, a maioria não consumia álcool $(72,9 \%)$ e não fumava $(98,8 \%)$. Quanto aos motivos pela procura de atendimento nutricional, foi observado que a maioria dos pacientes procurou atendimento para redução de peso $(27,05 \%)$. O atendimento ambulatorial da clínica escola é fundamental para a população, pois pode proporcionar melhor qualidade de vida através de ações de educação nutricional, visando à prevenção precoce de doenças crônicas não transmissíveis.

Palavras-chave: Prontuários. Ambulatório. Doenças Crônicas não Transmissíveis.

\section{ABSTRACT}

The increasing number of chronic non-communicable diseases in the population is evident. Thus, the demand for nutritional care has increased. The study aimed to identify the profile of patients seen at the nutrition clinic of a school clinic in a private University in São Luís - MA. This is a cross-sectional study with secondary data collection that took place from August to September 2019. 85 medical records from the year 2018 of patients of both sexes and all ages were analyzed, and medical records with incomplete information pertinent to conducting the research. The data were transcribed into a previously prepared protocol. Of the 85 medical records evaluated, it was found that $54.1 \%$ of the patients were adults. The greatest demand for nutritional care was female (74.1\%). According to lifestyle, most did not consume alcohol (72.9\%) and did not smoke $(98.8 \%)$. As for the reasons for seeking nutritional care, it was observed that most patients sought care for weight reduction $(27.05 \%)$. The outpatient care of the school clinic is essential for the population, as it can provide a better quality of life through nutritional education actions, aiming at the early prevention of chronic noncommunicable diseases.

Key-words: Medical records. Ambulatory. Noncommunicable Chronic Diseases. 


\section{INTRODUÇÁO}

Nos últimos anos, é evidente a grande mudança no perfil nutricional da sociedade, em que a expectativa de vida, a redução de mortes por doenças infecciosas e o aumento de óbitos por doenças crônicas não transmissíveis (DCNTs), alteraram o perfil de saúde das populações e o excesso de peso foi o principal fator (VIUDES et al., 2014).

De acordo com a Organização Pan-Americana de Saúde (OPAS), as doenças crônicas são responsáveis por 36 milhões de mortes no mundo, o que caracteriza $63 \%$ das mortes registradas no ano de 2018 (ONU, 2018).

No Brasil, as DCNTs são consideradas como um dos maiores problemas de saúde, representando $72 \%$ das causas de mortes, tendo como referência as doenças cardiovasculares, câncer, diabetes e doenças respiratórias crônicas (MALTA et al., 2015). A obesidade que é caracterizada como o excesso de gordura corporal, é o principal fator de risco para desenvolvimento destas doenças (PEREIRA et al., 2015), tendo origem pelo consumo excessivo de alimentos ultraprocessados, sedentarismo, fatores genéticos, socioculturais e psicossociais (VIUDES et al., 2014).

Segundo Demenech e Bernardes (2017), dieta e prática de atividade física fazem parte de uma prevenção primária contra o excesso de peso. Além disso, a orientação nutricional no sobrepeso e obesidade seria obter um balanço energético negativo, com variável déficit calórico e ingestão equilibrada de macronutrientes e micronutrientes, levando em conta a individualidade de cada um, considerando seus hábitos de vida, condições socioeconômicas, preferências alimentares e a manutenção da saúde.

Por isso, nos últimos anos, está crescendo a procura por atendimentos nutricionais, como forma de modificar os hábitos alimentares dos indivíduos, tornando esses hábitos mais saudáveis. Nesse sentido, a educação nutricional tem papel importante no atendimento nutricional, pois pode prevenir e controlar problemas relacionados a alimentação, oferecendo melhor qualidade de vida (MACEDO; AQUINO, 2018).

Nesse contexto, o objetivo desse estudo foi identificar o perfil dos pacientes atendidos no ambulatório de nutrição de uma Clínica escola em uma Universidade particular de São Luís - MA. 


\section{MATERIAIS E METODOS}

O estudo foi do tipo transversal com coleta de dados secundária, realizado no Ambulatório de Nutrição da Clínica Escola da Universidade Ceuma - Campus Renascença, São Luís - MA. A coleta de dados aconteceu no período de agosto a setembro de 2019.

Foram incluídos no estudo pacientes de ambos os sexos e de todas as idades (crianças, adolescentes, adultos e idosos) atendidos no ano de 2018.

A amostra foi do tipo não probabilística. Durante o ano de 2018 foram registrados um total de 92 prontuários, sendo analisados apenas 85 , pois 7 prontuários foram excluídos da pesquisa, em virtude de os mesmos conterem informações incompletas.

Os dados foram coletados dos prontuários de cada paciente, sendo transcritos para um protocolo que foi previamente elaborado, o qual possuía informações de data de atendimento, nome, idade, sexo, estado civil, data de nascimento, profissão, hábitos de vida (tabagismo, uso de álcool, prática de atividade física) e motivo da procura pelo atendimento.

O banco de dados foi organizado com o auxílio do Programa Microsoft Excel® 2010, para o desenvolvimento da análise estatística descritiva, utilizando-se as variáveis categóricas descritas por meio de suas frequências absolutas (n) e relativas (\%).

A pesquisa seguiu as recomendações da Resolução ํo 466 de 12 de dezembro de 2012 do Conselho Nacional de Saúde, bem como seus complementares, sendo aprovada pelo Comitê de Ética em Pesquisa da Universidade Ceuma sob o número de parecer 3.118.520.

\section{RESULTADOS E DISCUSSÄO}

$\mathrm{Na}$ tabela 1 encontram-se os resultados referentes às características sociodemográficas e hábitos de vida dos 85 prontuários dos pacientes analisados, $74,1 \%$ $(n=63)$ eram do sexo feminino e $25,9 \%(n=22)$ eram do sexo masculino e a maior prevalência dos atendimentos ambulatoriais foi de adultos, na faixa etária entre $\geq 20$ e $<60$ anos $(54,1 \%)$, seguido pelos idosos $(40 \%)$. 
Tabela 1. Características sociodemográficas e hábitos de vida de pacientes atendidos em uma Clínica escola de uma Universidade particular. São Luís, 2018.

\begin{tabular}{|c|c|c|}
\hline Variáveis & $\mathbf{n}$ & $\%$ \\
\hline \multicolumn{3}{|l|}{ Sexo } \\
\hline Feminino & 63 & 74,1 \\
\hline Masculino & 22 & 25,9 \\
\hline \multicolumn{3}{|l|}{ Faixa etária } \\
\hline$<10$ & 3 & 3,5 \\
\hline$\geq 10$ e $<20$ & 2 & 2,4 \\
\hline$\geq 20$ e $<60$ & 46 & 54,1 \\
\hline$\geq 60$ & 34 & 40 \\
\hline \multicolumn{3}{|l|}{ Estado civil } \\
\hline Solteiro & 41 & 48,3 \\
\hline Casado & 28 & 32,9 \\
\hline Viúvo & 15 & 17,6 \\
\hline Divorciado & 1 & 1,2 \\
\hline \multicolumn{3}{|l|}{ Tabagismo } \\
\hline Sim & 1 & 1,2 \\
\hline Não & 84 & 98,8 \\
\hline \multicolumn{3}{|c|}{ Bebida Alcóolica } \\
\hline Sim & 23 & 27,1 \\
\hline Não & 62 & 72,9 \\
\hline \multicolumn{3}{|c|}{ Exames laboratoriais } \\
\hline Sim & 23 & 27,1 \\
\hline Não & 62 & 72,9 \\
\hline \multicolumn{3}{|c|}{ Encaminhamento } \\
\hline Sim & 57 & 67,1 \\
\hline Não & 28 & 32,9 \\
\hline \multicolumn{3}{|c|}{ Atividade Física } \\
\hline Praticam & 30 & $35,3 \%$ \\
\hline Não praticam & & $64,7 \%$ \\
\hline \multicolumn{3}{|l|}{ Frequência } \\
\hline $1-2$ vezes & 10 & $33,3 \%$ \\
\hline $3-4$ vezes & 14 & $46,7 \%$ \\
\hline$>4$ vezes & 6 & $20 \%$ \\
\hline
\end{tabular}

Fonte: Autores, 2019.

A faixa etária prevalente desse estudo corrobora com o encontrado na pesquisa de De Souza et al. (2017), onde 63,7\% dos pacientes atendidos em um ambulatório de nutrologia de Ribeirão Preto eram adultos e 8,5\%idosos. No estudo de Brasil et al. (2018), 
realizado no ambulatório de nutrição de uma faculdade particular do Recôncavo Baiano, foi observado uma maior prevalência de adultos jovens (60,78\%) entre 18 e 39 anos, enquanto os idosos tiveram menor prevalência (8,23\%).Na pesquisa de Saraiva et al. (2014), realizado em um ambulatório de nutrição de uma universidade do Rio Grande do Sul, a maioria dos pacientes eram adultos $(73,83 \%)$ e $8,41 \%$ eram idosos.

Os achados do presente estudo corroboram com a pesquisa de Demenech e Bernardes (2017), na qual houve o predomínio de atendimento das mulheres $(88,4 \%)$ na clínica escola de uma Instituição de Ensino Superior do Vale do Rio dos Sinos, com média de idade de 47,9 anos $\pm 13,9$. Em estudo de Rodrigues e Silveira (2015), realizado no ambulatório da Universidade Federal de Goiás (UFG), 91,1\% eram mulheres.

De acordo com Viudes et al. (2014), a procura maior das mulheres pelo atendimento nutricional, pode ser devido ao maior acesso à informação e ao cuidado com a saúde e estética apresentado por esse grupo.

Em relação ao estado civil encontrado, houve um maior percentual de pacientes que se encontravam solteiros $48,3 \%$ ( $n=41)$ no dia do atendimento, seguido por $32,9 \%$ ( $n=28)$ casados, $17,6 \%(n=15)$ viúvos e o menor percentual encontrado foi dos divorciados $1,2 \%$ $(n=1)$ (Tabela 1), diferente da pesquisa de Brasil et al. (2018), na qual a maior prevalência foi de pacientes casados (52\%) seguido de solteiros (36\%).

A maioria dos pacientes (98,8\%) não possuía o hábito de fumar (Tabela 1). Esses dados corroboram com a pesquisa de Pfaffenseller et al. (2017), realizado em uma Clínica escolade nutrição em Salvador - Bahia, onde verificou-se que a maioria $(96,2 \%)$ dos pacientes atendidos não fumavam, isso demonstra um grande impacto positivo, visto queo hábito de fumar pode trazer consequências futuras à saúde. De acordo com o Instituto Nacional do Câncer, o tabagismo é responsável por 90\% dos cânceres de pulmão, além de ser fator de risco importante para AVCs (acidentes vasculares cerebrais) e ataques cardíacos fatais (INCA, 2018).

Quanto ao hábito do consumo de bebidas alcoólicas, grande parte dos pacientes (72,9\%) afirmou não possuir o hábito de ingeri-las (Tabela 1), o que representa fator positivo para uma melhor qualidade de vida. É importante frisar que o consumo nocivo de álcool está relacionado com lesões resultantes de acidentes de trânsito e violências interpessoais, distúrbios digestivos, doenças cardiovasculares, câncer, transtornos mentais e outras doenças (OPAS, 2018).

De acordo com os dados coletados neste estudo, a maioria $(64,7 \%)$ dos pacientes não realizavam nenhum tipo de atividade física (Tabela 1). No estudo de Silva et al. (2018), 
realizado com pacientes atendidos no ambulatório de nutrição de um Hospital Universitário de Maceió - Alagoas, observou-se que 67,14\% dos pacientes atendidos eram sedentários.

Na pesquisa de Saccon et al. (2015), 68,9\% dos pacientes atendidos no ambulatório de nutrição da Universidade Federal de Pelotas (UFPEL) não praticavam atividade física. Observa-se, que esses indivíduos possuem baixo nível de atividade física. Segundo Gonçalves (2017), níveis insuficientes de atividade física favorecem o desenvolvimento de doenças crônicas não transmissíveis, entre outras patologias.

Dos pacientes que praticavam atividade física, neste estudo prevaleceu à frequência 3-4 vezes por semana (46,7\%) (Tabela 1). A Organização Mundial da Saúde (OMS) ressalta que 1,4 bilhão de pessoas no mundo realizam atividades físicas insuficientes e no Brasil $47 \%$ da população é sedentária, isso aumenta os riscos para doenças crônicas não transmissíveis (ONU, 2018).

No presente estudo, a maioria dos pacientes (72,9\%) não apresentavam exames laboratoriais na primeira consulta (Tabela 1). Esses resultados corroboram com os resultados de Mendes e Lemos (2018) em que a maioria dos pacientes (66,5\%) atendidos no ambulatório do Centro Universitário Toledo em Araçatuba - São Paulo, não apresentaram exames laboratoriais, o que difere do estudo de Martins e Bortolini (2018), que a maioria (72\%) dos pacientes atendidos no ambulatório do Hospital de Guarnição de Bagé apresentou exames laboratoriais.

A presença de exames laboratoriais contribui para um atendimento mais eficaz. Com isso, há maior possibilidade de uma intervenção precoce (GOMES; SALLES, 2010).

Os dados mostram que $67,1 \%$ dos pacientes foram para consulta ao nutricionista através de encaminhamento médico de outros profissionais (Tabela 1). É possível perceber por meio do encaminhamento que, o que motivou esses indivíduos a procurarem o serviço de nutrição, foi pela existência de algum tipo de DCNT (doenças crônicas não transmissíveis) instalada (Tabela 2). 
Tabela 2. Motivos pela procura do atendimento nutricional de pacientes atendidos em uma Clínica escola de uma Universidade particular. São Luís, 2018.

(continua)

\begin{tabular}{lcc}
\hline Variável & $\mathbf{n}$ & \% \\
\hline Motivo da procura & 1 & 1,2 \\
Dislipidemia & 3 & 3,5 \\
Diabetes Mellitus & 6 & 7,05 \\
HAS & 23 & 27,05 \\
Redução de peso/Sobrepeso/Obesidade & 22 & 26 \\
Síndrome Metabólica & 14 & 16,5 \\
Doenças do TGI & & \\
\hline & & $\mathbf{2 , 3 5}$ \\
Constipação & $\mathbf{2}$ & $\mathbf{1 , 2}$ \\
Diarréia & $\mathbf{1}$ & $\mathbf{3 , 5}$ \\
Ganhar peso & $\mathbf{3}$ & $\mathbf{3 , 5}$ \\
Reeducação alimentar & $\mathbf{3}$ & $\mathbf{2 , 3 5}$ \\
Doenças do TGI + Obesidade & $\mathbf{2}$ & $\mathbf{1 , 2}$ \\
Esteatose hepática + Dislipidemia & $\mathbf{1}$ & $\mathbf{1 , 2}$ \\
Esteatose hepática + Obesidade & $\mathbf{1}$ & $\mathbf{2 , 3 5}$ \\
Esteatose hepática + Doenças do TGI & $\mathbf{2}$ & $\mathbf{1 , 2}$ \\
Ansiedade + HAS & $\mathbf{1}$ &
\end{tabular}

Fonte: Autores, 2019.

TGI: trato gastrointestinal; HAS: hipertensão arterial sistêmica.

De acordo com a Tabela 2, foi verificado que a maioria dos pacientes $(27,05 \%)$ procurou atendimento nutricional para redução de peso. Na pesquisa de Azevedo et al. (2014), realizada com funcionários da área da saúde de uma universidade pública em Recife, observou-se que $60 \%$ encontrava-se em excesso de peso, e no estudo de Oliveira et al. (2015), 83,3\% dos pacientes atendidos no Centro de Saúde Escola em São Paulo estavam com excesso de peso ou obesidade.

Santos Gularte (2018) afirma que o sobrepeso e a obesidade são considerados problemas de saúde pública e epidemias globais. A obesidade cresceu de 11,8\% em 2006 para 18,6\% em 2016 e contribui para prevalência de hipertensão arterial e diabetes. $\mathrm{O}$ excesso de peso também aumentou de 42,6\% em 2006 para 53,8\% em 2016 (MINISTÉRIO DA SAÚDE, 2017). Uma má alimentação e atividade física insuficiente são importantes fatores de risco para a obesidade (FERREIRA; SZWARCWALD; DAMACENA, 2019).

A segunda maior prevalência de procura por atendimento foi devido à presença de síndrome metabólica, presente em $26 \%$ dos pacientes (Tabela 2). A Síndrome metabólica está associada a um conjunto de fatores de risco metabólicos, que têm como base a resistência à insulina, sendo estes fatores a obesidade central, hipertensão arterial sistêmica, glicemia alterada, triglicerídeo elevado e baixo HDL (high density lipoprotein) 
(SOCIEDADE BRASILEIRA DE ENDOCRINOLOGIA, 2008). No estudo de Pinho et al. (2014), realizado no ambulatório de nutrição de um Hospital Universitário em Belém - Pará, a maioria $(72,86 \%)$ dos pacientes atendidos possuíam síndrome metabólica (SM), destes $66,67 \%$ eram mulheres.

A presença de doenças do trato gastrointestinal, como gastrite, refluxo, doença de Crohn, síndrome dispéptica e pirose, também foi outro motivo relevante de procura por atendimento nutricional por $16,5 \%$ dos pacientes (Tabela 2). Na pesquisa de De Maman Oldra et al. (2017), 13,5\% dos pacientes atendidos em uma clínica escola de nutrição da região sudoeste do Paraná possuíam problemas do trato gastrointestinal. No estudo de Saccon et al. (2015), foi verificado que as doenças do trato gastrointestinal estavam associadas a outras patologias em $52 \%$ dos pacientes atendidos. De acordo com os dados do presente estudo, houve uma baixa prevalência de doenças do trato gastrointestinal associadas a outras patologias.

\section{CONSIDERAÇÕES FINAIS}

Diante da análise dos resultados, identificou-se que a maioria dos pacientes atendidos no ambulatório de nutrição, foi de indivíduos adultos e do gênero feminino. Foi observado que hábitos de vida, como consumo de bebidas alcoólicas, tabagismo e prática de atividades físicas, tiveram baixo percentual nesta população. Verificou-se ainda que um dos principais motivos da procura pelo atendimento nutricional foi à busca pela redução de peso.

Portanto, o atendimento ambulatorial da clínica escola é fundamental para a saúde da população, pois pode proporcionar melhor qualidade de vida a essas pessoas através de ações de educação nutricional, visando a prevenção precoce de doenças crônicas não transmissíveis.

O presente estudo colaborou com a temática abordada contribuindo assim para 0 conhecimento do perfil do público atendido e, consequentemente, um melhor atendimento ambulatorial. 
AZEVEDO, E. C. C. et al. Consumo alimentar de risco e proteção para as doenças crônicas não transmissíveis e sua associação com a gordura corporal: um estudo com funcionários da área de saúde de uma universidade pública de Recife (PE), Brasil. Ciência \& Saúde Coletiva, v. 19, n. 5, p. 1613-1622, 2014.

DEMENECH, M. C.; BERNARDES, S. Metas alimentares versus dieta: qual oferece melhores resultados em pacientes com excesso de peso?.Revista da Associação Brasileira de Nutrição-RASBRAN, v. 8, n. 1, p. 26-30, 2017.

BRASIL, Aíla Cassiana Gomes. Perfil nutricional dos pacientes do Ambulatório de Nutrição de uma faculdade privada do Recôncavo Baiano. 2018. Trabalho de Conclusão de Curso (Bacharelado em Nutrição) - Faculdade Maria Milza, Bahia, 2018.

DE MAMAN OLDRA, C. et al. Ingestão de macronutrientes e colesterol por pacientes atendidos em uma clínica-escola de nutrição. Biosaúde, v. 18, n. 2, p. 64-74, 2017.

DE SOUZA, A. C. B. et al. Perfil dos pacientes obesos no primeiro atendimento em Ambulatório de Nutrologia Municipal de Ribeirão Preto (SP). Medicina (Ribeirão Preto, Online.), v. 50, n. 4, p. 207-15, 2017.

FERREIRA, A. P. S.; SZWARCWALD, C. L.; DAMACENA, G.N. Prevalência e fatores associados da obesidade na população brasileira: estudo com dados aferidos da Pesquisa Nacional de Saúde, 2013. Revista Brasileira de Epidemiologia, v. 22, p. e190024, 2019.

GOMES, A. C. R.; SALLES, D. R. M. Perfil nutricional dos pacientes atendidos no ambulatório de Nutrição da Faculdade de Ciências da Saúde (FACISA), de Patos de Minas/MG. Perquirere: Revista do Núcleo Interdisciplinar de Pesquisa e Extensão do UNIPAM, v. 1, n. 7, p. 63-71, 2010.

GONÇALVES, E. C. A. et al. Baixos níveis de atividade física em servidores públicos do sul do Brasil: associação com fatores sociodemográficos, hipercolesterolemia e diabetes. Revista Andaluza de Medicina del Deporte, v. 10, n. 2, p. 54-59, 2017.

INCA, INSTITUTO NACIONAL DE CÂNCER. Tabagismo. Disponível em: https://www.inca.gov.br/tabagismo. Acesso em: 08 de nov. 2019.

MACEDO, I. C.; AQUINO, R. C. "O Marco de Referência de Educação Alimentar e Nutricional para Políticas Públicas" no Brasil no contexto do atendimento nutricional. Demetra: Alimentação, Nutrição \& Saúde, v. 13, n. 1, p. 21-35, 2018.

MALTA, D. C. et al. A vigilância e o monitoramento das principais doenças crônicas não transmissíveis no Brasil-Pesquisa Nacional de Saúde, 2013. Revista Brasileira de Epidemiologia, v. 18, n. suppl 2, p. 3-16, 2015.

MARTINS, A.; BORTOLINI, V. Perfil de pacientes que procuram atendimento no Ambulatório de Nutrição do Hospital de Guarnição de Bagé (HGUBA). Revista Congrega - Mostra de Trabalhos de Conclusão de Curso - ISSN 2595-3605, n. 2, p. 610-625, 2018. 
MENDES, S. D.; LEMOS, A. C. G. Perfil nutricional dos pacientes atendidos no Ambulatório de Nutrição do Centro Universitário Toledo, Araçatuba/SP. 2018. Trabalho de Conclusão de Curso (Bacharelado em Nutrição) - Centro Universitário Toledo, Araçatuba, 2018.

MINISTÉRIO DA SAÚDE. Em dez anos, obesidade cresce $60 \%$ no Brasil e colabora para maior prevalência de hipertensão e diabetes. Disponível em: http://www.saude.gov.br/noticias/agencia-saude/28108-em-dez-anos-obesidade-cresce60-no-brasil-e-colabora-para-maior-prevalencia-de-hipertensao-e-diabetes. Acesso em: 08 de nov. 2019.

OLIVEIRA, M. C. et al. Aplicação do Índice de Qualidade da Dieta Revisado antes e após orientação nutricional. Revista de Ciências Médicas, v. 24, n. 1, p. 29-36, 2015.

ONU, ORGANIZAÇÃO DAS NAÇÕES UNIDAS. Doenças crônicas são responsáveis por $63 \%$ de todas as mortes no mundo. Disponível em: https://nacoesunidas.org/doencascronicas-sao-responsaveis-por-63-de-todas-as-mortes-no-mundo-diz-opas/. Acesso em: 10 de mar. 2019.

ONU, ORGANIZAÇÃO DAS NAÇÕES UNIDAS. Mais de 1,4 bilhão de adultos no mundo não praticam atividade física suficiente. Disponível em: https://nacoesunidas.org/maisde-14-bilhao-de-adultos-no-mundo-nao-praticam-atividade-fisica-suficiente/. Acesso em: 08 de nov. 2019.

OPAS, ORGANIZAÇÃO PAN - AMERICANA DA SAÚDE. Uso nocivo de álcool mata mais de 3 milhões de pessoas a cada ano; homens são a maioria. Disponível em: https://www.paho.org/bra/index.php?option=com_content\&view=article\&id=5763:usonocivo-de-alcool-mata-mais-de-3-milhoes-de-pessoas-a-cada-ano-homens-sao-amaioria\&Itemid=839/. Acesso em: 08 de nov. 2019.

PEREIRA, M. A. et al. Desafios e reflexões na implantação de um programa de Educação Alimentar e Nutricional (EAN) em indivíduos com excesso de peso. Revista Brasileira em Promoção da Saúde, v. 28, n. 2, p. 290-296, 2015.

PFAFFENSELLER, R. F. et al. Perfil sociodemográfico, comportamental e nutricional de adultos atendidos em uma Clínica-escola de Nutrição em Salvador, Bahia. Revista de Ciências Médicas e Biológicas, v. 16, n. 3, p. 380-386, 2017.

PINHO, P. M. de et al. Síndrome metabólica e sua relação com escores de risco cardiovascular em adultos com doenças crônicas não transmissíveis. Revista da Sociedade Brasileira de Clínica Médica, v. 12, n. 1, p. 22-30, 2014.

RODRIGUES, A. P. S.; SILVEIRA, E. A. Correlação e associação de renda e escolaridade com condições de saúde e nutrição em obesos graves. Ciência \& Saúde Coletiva, v. 20, n. 1, p. 165-174, 2015.

SACCON, T. et al. Perfil e evolução do estado nutricional de pacientes que frequentam um ambulatório de nutrição do Sul do Brasil. Nutrición clínica y dietética hospitalaria, v. 35, n. 3, p. 74-82, 2015. 
SANTOS GULARTE, Laura et al. Perfil, estado nutricional e variação de peso de mulheres adultas atendidas em um ambulatório de nutrição. Nutricion Clinica y Dietetica Hospitalaria, v. 38, n. 4, p. 33-38, 2018.

SARAIVA, D. I.; ZEMOLIN, G. P.; ZANARDO, V. P. S. Perfil Nutricional de Pacientes Atendidos em um Ambulatório de Especialidades em Nutrição. Revista Vivências, v. 10, n. 19, p. 113-121, 2014.

SBEM, SOCIEDADE BRASILEIRA DE ENDOCRINOLOGIA E METABOLOGIA. Síndrome Metabólica. Disponível em: https://www.endocrino.org.br/sindrome-metabolica/. Acesso em: 08 de nov. de 2019.

SILVA, M. B. G. et al. Perfil clínico e nutricional dos indivíduos atendidos em um ambulatório de nutrição do hospital universitário (HUPAA/UFAL). Gep News, v. 1, n. 1, p. 139-144, 2018.

VIUDES, D. R. et al. Perfil nutricional e consumo alimentar de pacientes com excesso de peso atendidos por um ambulatório de nutrição. Publicatio UEPG: Ciências Biológicas e da Saúde, v. 20, n. 2, p. 115-124, 2015. 